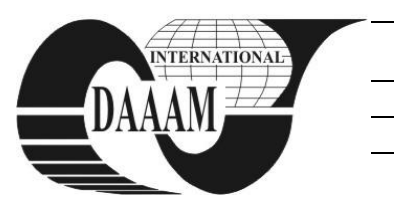

\title{
GENETIC ALGORITHM OPTIMIZATION OF A SHIP'S BULBOUS BOW
}

\author{
MATULJA, D[unja] \& DEJHALLA, R[oko]
}

\begin{abstract}
A genetic algorithm technique coupled with a potential flow solver has been applied to deal with the hydrodynamic optimization of a bulbous ship hull form. A non interactive procedure has been developed to minimize the objective function, related to the flow past a ship moving with steady forward speed in calm water. A Bézier surface, defined by several bulb geometrical parameters, has been used to model the forebody and to allow the modifications. According to the geometrical constraints, a new optimized hull form has been automatically obtained. The hull form with the obtained modification of the bulb has been presented and analyzed in order to demonstrate the effectiveness and validity of the developed procedure.
\end{abstract}

Key words: genetic algorithm, hydrodynamic optimization

\section{INTRODUCTION}

The prediction of ship resistance is normally based on results from model tests at towing tanks. However, different computational tools can be used for preliminary selection of variants before testing, as well as to study flow details to gain insight into how a ship hull form can be improved. Mathematically, the optimization problem can be formulated as the determination of a set of design variables subjected to certain relations between variables and restrictions of these variables. From the hydrodynamic point of view, the most interesting optimization contribution is to minimize the ship resistance, or the ship resistance components. Many interesting works on hull form optimization have been presented through the years (Peri et al., 2000, Percival et al., 2001, Campana et al., 2006, Wilson et al., 2011).

In the paper, numerical optimization has been applied to the S-175 containership hull form. Two independent optimization procedures have been performed with different objective functions: in one it was set as the lowest root sum square (RSS) of wave elevations in the bow area, while in the other as the lowest wave resistance. The developed procedure proved to be effective, and the idea is to enhance the potential flow solver to obtain a nonlinear potential flow solution for more accurate results in optimization of the hulls with bulbous bow.

\section{OPTIMIZATION SETUP}

The optimization problems may be generally formulated as problems of minimizing an objective function $\mathrm{f}(\boldsymbol{\xi})$, of a number of variables $\xi_{1}, \xi_{2}, \xi_{3}, \ldots, \xi_{n}$ subject to a group of constraints that can be formulated as equalities or inequalities. The solution of the optimization problem calls for the formulation of a suitable optimization procedure. Therefore, the three-dimensional potential flow solver (Dejhalla, 1999) and the genetic algorithm (Goodman, 1996) have been coupled to build a procedure for the bulbous bow optimization. The optimization procedure starts from a specified basis hull form, by calculating the flow past the hull form. The genetic algorithm creates an initial population of specified number of individuals (hull shapes), randomly generated within upper and lower bounds for the design variables. The bow geometry modification algorithm is an integral component of the optimization procedure. It allows to obtain the changes of the bulb shape and to automatically remesh the fore part of the hull form for each design case. As geometrical constraints, the design waterline and the stem profile were kept the same as in the basis form, while the shape of the fore part was allowed to change only by altering the $y-$ coordinates of the chosen points. In this manner, $y_{1}, y_{2}, y_{3}, \ldots, y_{n}$ form the set of design variables, taken as variables $\xi_{1}, \xi_{2}, \xi_{3}, \ldots$, $\xi_{n}$ introduced previously. The number of design variables $n$ treated in the optimization procedure must remain within some reasonable range. The following genetic algorithm parameters have been adopted:

- String length $=7$

- Crossover probability $\mathrm{p}_{\mathrm{c}}=0.5$

- Mutation probability $\mathrm{p}_{\mathrm{m}}=0.3$

- Population size $=40$

- $\quad$ Number of generations $=50$

As objective function, two approaches were carried out. For the first, the objective function was set as the wave resistance:

$$
\mathrm{f}(\xi)=R_{\mathrm{W}}
$$

For the other approach, the RSS of the wave elevation in the bow area has been defined as follows:

$$
\mathrm{f}(\xi)=\sqrt{\sum_{i} \eta_{i}^{2}},
$$

where $\eta_{\mathrm{i}}$ is the wave elevation for the $i$-th free surface panel located in the circular area of the free surface with the center on the fore perpendicular and the radius equal to $30 \% L_{\mathrm{PP}}$.

In both cases the target was to obtain the minimum objective function. The wave elevations and the wave resistance values are computed by the potential flow solver (Dejhalla, 1999).

\section{BÉZIER SURFACE}

In order to allow the modifications of the bulbous bow defined by several parameters, the fore part has been modeled with a Bézier surface because of its possibility to create a grid of desired density from a relatively low number of points. The Bézier surface is a species of mathematical spline used in computer graphics and computer-aided design. It consists of a patch defined by a set of control points, and it enables to generate a mesh of quadrilaterals of any required density. The Bézier surface passes through the four points which define the vertices of the patch, while it does not generally pass through the other control points. It is rather attracted by those points, creating a smooth surface which can adequately match the hull form surface. The obtained surface is mathematically convenient since it provides any required number of quadrilaterals and keeps good continuity properties. A Bézier surface of order $(n, m)$ needs a grid of $(n+1)(m+1)$ control 
points $P(i, j)$. If $p(u, v)$ is a function of the parametric coordinates $(u, v)$, then the Bézier surface can be defined as a parametric surface given by:

$$
p(u, v)=\sum_{i=0}^{n} \sum_{j=0}^{m} B_{i}^{n}(u) B_{j}^{m}(v) \cdot p_{i, j}
$$

In the above expression the Bernstein polynomials are expressed by:

$$
B_{i}^{n}(u)=\left(\begin{array}{c}
n \\
i
\end{array}\right) u^{i}(1-u)^{n-1}, B_{j}^{m}(v)=\left(\begin{array}{c}
m \\
j
\end{array}\right) v^{j}(1-v)^{m-1}
$$

where

$$
\left(\begin{array}{l}
n \\
i
\end{array}\right)=\frac{n !}{i !(n-i) !},\left(\begin{array}{c}
m \\
j
\end{array}\right)=\frac{m !}{j !(m-j) !}
$$

are the binomial coefficients.

\section{RESULTS}

The optimization procedure has been applied to the S-175 hull form taken as the basis hull form. The fore part of the hull form has been optimized for a single speed corresponding to the Froude number $F r=0.22$. The Froude number is based on $L_{\mathrm{PP}}$.

\begin{tabular}{|l|l|l|}
\hline Length between perpendiculars, $L_{\mathrm{PP}}$ & 175.0 & $\mathrm{~m}$ \\
\hline Breadth & 25.4 & $\mathrm{~m}$ \\
\hline Draught & 9.5 & $\mathrm{~m}$ \\
\hline Displacement & 24779.7 & $\mathrm{t}$ \\
\hline Block coefficient & 0.572 & \\
\hline Midship coefficient & 0.97 & \\
\hline
\end{tabular}

Tab. 1. Principal particulars of the basis hull form

The optimized bulb shapes are compared to the initial bow shape in Fig. 1. and Fig. 2. The results of the optimization procedure are evident. Furthermore, although the problem is treated as a single point design problem, additional numerical tests have been performed with the obtained hull form for Froude numbers ranging from 0.175 to 0.28 . The predicted wave resistance values are summarized in Tab. 2. and Fig. 3. It can be noticed that the optimization based on the wave elevations provides a certain improvement only for higher speeds, even though the RSS of the wave elevations is decreased. The optimization based on the wave resistance gives more satisfying results for this case.

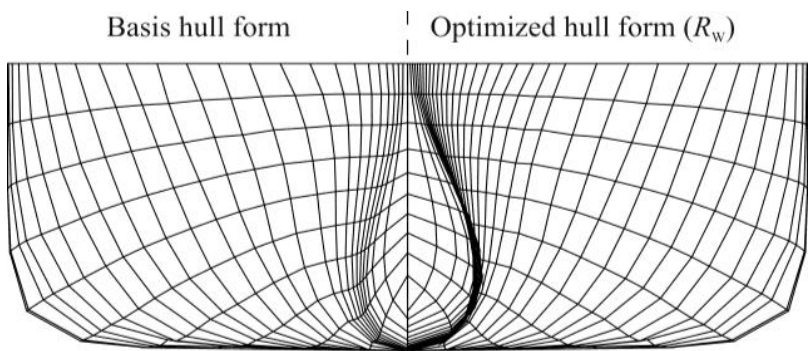

Fig. 1. Comparison of the basis and optimized hull form, optimization based on $R_{\mathrm{W}}$

Basis hull form Optimized hull form $\left(\eta_{\mathrm{i}}\right)$

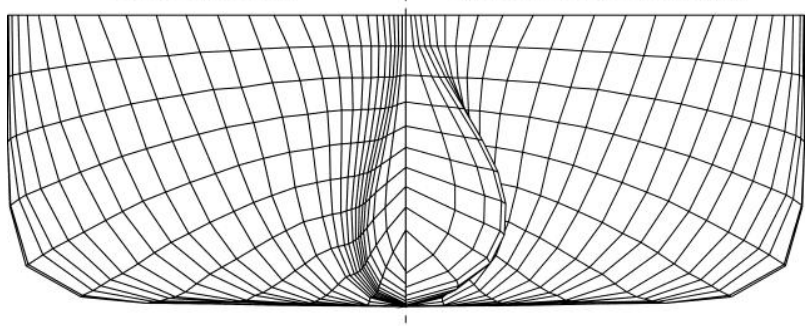

Fig. 2. Comparison of the basis and optimized hull form, optimization based on $\eta_{i}$

\begin{tabular}{|c|c|c|c|}
\cline { 2 - 4 } \multicolumn{1}{c|}{} & $\begin{array}{c}\text { Basis hull } \\
\text { form }\end{array}$ & $\begin{array}{c}\text { Optimization } \\
\text { based on } R_{\mathrm{W}}\end{array}$ & $\begin{array}{c}\text { Optimization } \\
\text { based on } \eta_{\mathrm{i}}\end{array}$ \\
\hline$F r$ & $R_{\mathrm{W}}, \mathrm{kN}$ & $R_{\mathrm{W}}, \mathrm{kN}$ & $R_{\mathrm{W}}, \mathrm{kN}$ \\
\hline 0.175 & 9.300 & 15.919 & 51.365 \\
\hline 0.20 & 21.810 & 16.117 & 39.799 \\
\hline 0.22 & 95.739 & 77.798 & 89.940 \\
\hline 0.24 & 129.739 & 106.670 & 114.160 \\
\hline 0.26 & 205.882 & 177.982 & 180.822 \\
\hline 0.28 & 353.063 & 317.829 & 316.510 \\
\hline
\end{tabular}

Tab. 2. Comparison of the results

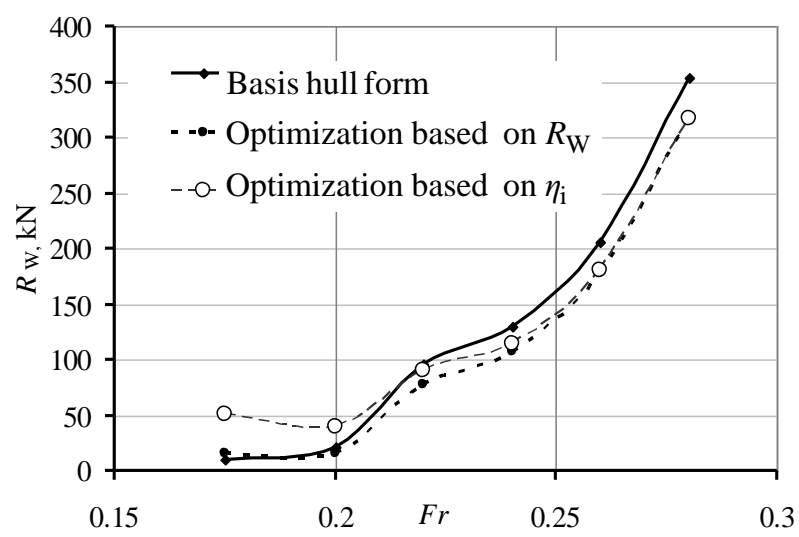

Fig. 3. Comparison of the wave resistances

\section{CONCLUSION}

The study has shown that the developed optimization procedure can be successfully applied to the optimization of the ship hull form and used as a valuable method to favorably modify the fore part. In the optimization procedure, different objective functions may be considered and as a consequence different outcomes can be expected. In order to avoid multiple solutions, a procedure that involves multi-objective optimization will be developed in the future, and a wider range of design variables will be enabled. This way the changes in the stem profile will also be allowed, so a wider variety of bulb shapes is expected to be generated. In order to model more effectively the flow past the forebody, a nonlinear potential flow solver will be implemented.

\section{REFERENCES}

Campana, E. F., Peri, D., Tahara, Y. \& Stern, F. (2006). Shape optimization in ship hydrodynamics using computational fluid dynamics. Computer Methods in Applied Mechanics and Engineering, Vol 196, pp. 634-651. Elsevier

Dejhalla, R. (1999). Numerical Modeling of Flow Around the Ship Hull, Ph.D. Thesis. Rijeka, Faculty of Engineering, University of Rijeka

Goodman, E.D. (1996). An Introduction to GALLOPS - The "Genetic Algorithm Optimized for Portability and Parallelism" System, Release 3.2, Technical Report \#9607-01, East Lansing : Michigan State University.

Percival, S., Hendrix, D. \& Noblesse, F. (2001). Hydrodynamic optimization of ship hull forms, Applied Ocean Research, Vol 23, pp. 337-355. Elsevier

Peri, D., Rossetti, M. \& Campana, F. (2000). Improving the hydrodynamic characteristics of ship hulls via numerical optimization techniques. Proceedings of IX Congress IMAM 2000, pp. 16-24. Ischia

Wilson, W., Hendrix, D. \& Gorski, J. (2011). Hull form optimization for early stage ship design, Naval Engineers Journal 122: 53-65. Alexandria 